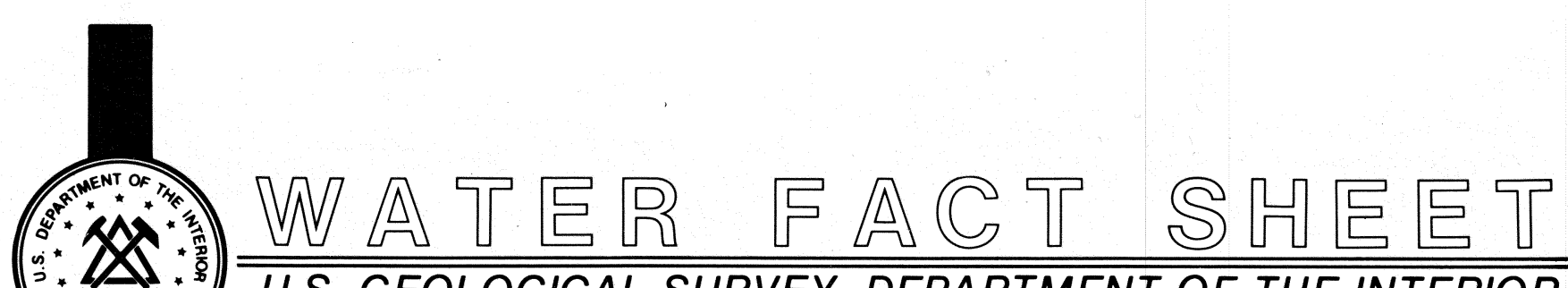

U.S. GEOLOGICAL SURVEY, DEPARTMENT OF THE INTERIOR

\title{
LARGEST RIVERS IN THE UNITED STATES
}

This fact sheet shows the location and ranking of the 20 largest rivers in the United States. It is common knowledge that the Mississippi is the largest U.S. river, but what is the rank of other major U.S. rivers? Rivers are considered large on the basis of one or more of three characteristics: total length from source to mouth, area of basin (watershed) drained by the stream, and average rate of flow (discharge) at the mouth. The alphabetical list on the back of this sheet shows these characteristics of 32 rivers so as to include the 20 largest rivers in each of the three categories. Among the 32 rivers, 16 are tributary to other rivers on the list; the remaining rivers discharge directly into oceans, seas, gulfs, or bays.

As dynamic parts of our environment, rivers and their characteristics vary in space and time in response to climatic changes and to man's activities. The causes include seasonal and annual changes in precipitation and temperature, cycles of erosion and deposition (especially during floods), diversions of water (for irrigation, power, and other purposes), and the construction of public works-dams, levees, locks, and canals. For example, combinations of these effects, but principally diversions, have reduced the average flow of the Colorado River near its mouth from about 22,000 cubic feet per second $\left(\mathrm{ft}^{3} / \mathrm{s}\right)$ for the period 1903-34 to less than $4,000 \mathrm{ft}^{3} / \mathrm{s}$ during the period 1951-80. However, the annual flow in 1984 averaged 17,500 $\mathrm{ft}^{3} / \mathrm{s}$, a consequence of record-breaking precipitation on the river basin. A flow of $1,000 \mathrm{ft}^{3} / \mathrm{s}$ is equal to 646 million gallons per day, 724,000 acre-feet per year, or 28.3 cubic meters per second. (One acre-foot is the volume of water that would cover 1 acre to a depth of 1 foot.)
River lengths or river-length data are affected not only by some of the natural and artificial causes noted in the preceding paragraph, but also by the precision of various techniques of measurement, by the scale of available maps or aerial photographs, and by somewhat arbitrary decisions. For example, the length may be considered to be the distance from the mouth to the most distant headwater source (irrespective of stream name) or from the mouth to the headwaters of the stream commonly identified as the source stream. The names of some rivers, such as the Mississippi River and the Rio Grande, are unchanged from source to mouth. In contrast, the name of the source of the Mobile River-Tickanetley Creek-changes five times before becoming Mobile River 45 miles north of Mobile Bay. The lengths of meandering rivers, such as the Mississippi River south of Cairo, Ill., undergo significant changes in length from time to time because of a natural or excavated cutoff (a channel severing a narrow strip of land, thus bypassing a large bend in a river) that reduces river length and therefore navigation time. For example, between 1766 and 1885, the length of the Mississippi River from Cairo, Ill., to New Orleans, La., was reduced by 218 miles because of 18 cutoffs (Elliott, 1932, page 59).

Reference cited-Elliott, D.O. (U.S. Mississippi River Commission), 1932, The improvement of the lower Mississippi River for flood control and navigation: Vicksburg, Miss., U.S. Waterways Experiment Station, U.S. Army Corps of Engineers, 345 pages.

For additional information write to:

U.S Geological Survey

Water Resources Division

MS 419, National Center

Reston, Virginia 22092

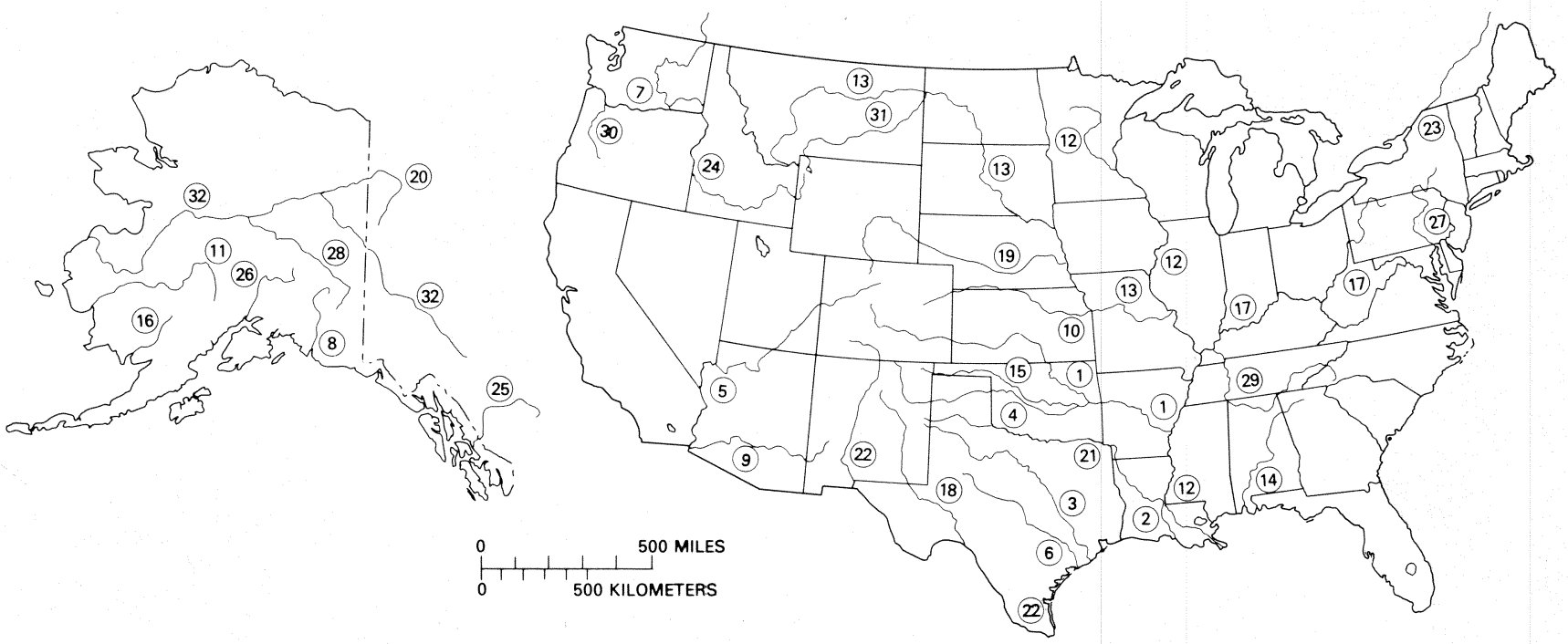

Maps showing location of largest rivers in the United States. 


\section{LARGEST RIVERS IN THE UNITED STATES, IN DISCHARge, DRAINAGE AREA, OR LENGTH}

[Of the 32 rivers listed here, the 20 largest in three categories - discharge, drainage basin, and length-are ranked from 1 to 20 ; these ranks are shown in parentheses. Abbreviations: $\mathrm{ft}^{3} / \mathrm{s}=$ cubic feet per second; $\mathrm{mi}^{2}=$ square miles. All data have been rounded to no more than three significant figures. Sources of data: Stream discharge and drainage area-mainly U.S. Geological Survey reports and files; length-publications and files of U.S. Geological Survey, U.S. Army Corps of Engineers, U.S. Environmental Protection Agency, and the Tennessee Valley Authority; data for the St. Lawrence River from "Facts from Canadian Maps," Canada Department of Energy, Mines and Resources, 1972. Period of record for most rivers is 1951-80. Some data are provisional and subject to revision. Compiled by J.C. Kammerer, U.S. Geological Survey]

NOTE: Rank from 1 to 20 in each category is shown in parentheses.

\begin{tabular}{|c|c|c|c|c|c|c|c|c|c|}
\hline \multirow{2}{*}{$\begin{array}{l}\begin{array}{l}\text { Number } \\
\text { on map }\end{array} \\
1 \ldots \ldots \ldots \ldots \ldots . . . . .\end{array}$} & \multirow[b]{2}{*}{ Arkansas .... } & \multirow[b]{2}{*}{ 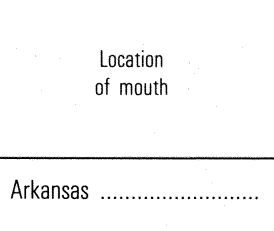 } & \multicolumn{2}{|c|}{$\begin{array}{c}\text { Average } \\
\text { discharge } \\
\text { at mouth } \\
\left|1,000 \mathrm{ft}^{3} / \mathrm{s}\right|\end{array}$} & \multicolumn{2}{|c|}{$\begin{array}{l}\text { Drainage area } \\
\left(1,000 \mathrm{mi}^{2}\right)\end{array}$} & \multicolumn{2}{|c|}{$\begin{array}{l}\text { Length } \\
\text { from } \\
\text { source to } \\
\text { mouth } \\
\text { (miles) }\end{array}$} & \multirow{2}{*}{$\begin{array}{l}\text { Source stream } \\
\text { (name and location) }\end{array}$} \\
\hline & & & 41.0 & $(16)$ & 161 & (9) & 1,460 & $(6)$ & \\
\hline $2 \ldots \ldots \ldots \ldots \ldots$ & $\begin{array}{l}\text { Atchafalaya lexcluding about } \\
167,000 \mathrm{ft}^{3} / \mathrm{s} \text { diverted from } \\
\text { Mississippi River). }{ }^{1}\end{array}$ & Louisiana ............................. & 58.0 & (11) & 95.1 & (11) & 1,420 & (8) & $\begin{array}{l}\text { Tierra Blanca Creek, New Mexico } \\
\text { (Curry County). }\end{array}$ \\
\hline $3 \ldots \ldots \ldots \ldots \ldots$ & 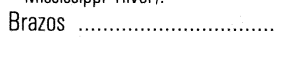 & 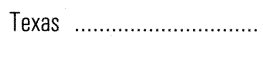 & $(*)$ & $\cdots$ & 45.6 & (19) & 1,280 & (111) & $\begin{array}{l}\text { Blackwater Draw, New Mexico } \\
\text { (Curry County). }\end{array}$ \\
\hline $4 \ldots \ldots \ldots \ldots \ldots . . . .$. & 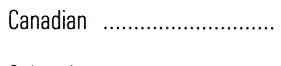 & 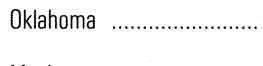 & $\left({ }^{*}\right)$ & $\cdots$ & 46.9 & (18) & 906 & $(16)$ & $\begin{array}{l}\text { Canadian River, Colorado } \\
\text { (Las Animas County). }\end{array}$ \\
\hline $5 \ldots \ldots \ldots \ldots \ldots$ & Colorado .............................. & Mexico ……..................... & $\left|{ }^{*}\right|$ & $\cdots$ & $\begin{array}{l}246 \\
\text { (U.S.-Mexico) }\end{array}$ & (7) & 1,450 & (7) & $\begin{array}{l}\text { Colorado River, Colorado } \\
\text { (Grand County). }\end{array}$ \\
\hline $6 \ldots \ldots \ldots \ldots \ldots$ & Colorado lof Texas) .............. & Texas …........................... & $\left({ }^{*}\right)$ & $\cdots$ & $\begin{array}{l}42.3 \\
4\end{array}$ & $\cdots$ & 862 & (18) & $\begin{array}{l}\text { Colorado River lof Texas), Texas } \\
\text { (Dawson County). }\end{array}$ \\
\hline $7 \ldots \ldots \ldots \ldots \ldots$ & Columbia .............................. & Oregon-Washington .......... & 265 & (4) & $\begin{array}{l}258 \\
\text { IU.S.-Canadal }\end{array}$ & (6) & 1,240 & (12) & $\begin{array}{l}\text { Columbia River, British Columbia } \\
\text { Canada. }\end{array}$ \\
\hline $8 \ldots \ldots \ldots \ldots \ldots$ & Copper ….............................. & 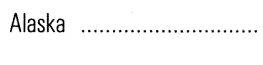 & 59 & $(10)$ & 24.4 & $\cdots$ & 286 & $\cdots$ & $\begin{array}{l}\text { Copper River at terminus of Copper } \\
\text { Glacier, Alaska. }\end{array}$ \\
\hline 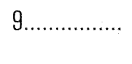 & 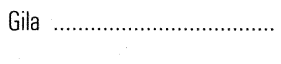 & 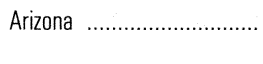 & $\left({ }^{*}\right)$ & $\cdots$ & $\begin{array}{l}58.2 \\
\text { (U.S.-Mexico) }\end{array}$ & (16) & 649 & $\cdots$ & $\begin{array}{l}\text { Middle Fork Gila River, New Mexico } \\
\text { (Catron County). }\end{array}$ \\
\hline $10 \ldots \ldots \ldots \ldots \ldots$ & 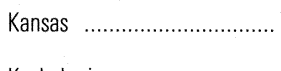 & 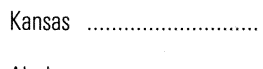 & $\left({ }^{*}\right)$ & $\cdots$ & 59.5 & (15) & 743 & $\cdots$ & $\begin{array}{l}\text { Arikaree River, Colorado } \\
\text { (Elbert County). }\end{array}$ \\
\hline $11 \ldots \ldots \ldots \ldots \ldots$ & Kuskokwim ........................... & Alaska ............................. & 67 & (9) & 48 & (17) & 724 & $\cdots$ & $\begin{array}{l}\text { South Fork Kuskokwim River at ter- } \\
\text { minus of unnamed glacier, Alaska. }\end{array}$ \\
\hline $12 \ldots \ldots \ldots \ldots . . . . .$. & $\begin{array}{l}\text { Mississippi lexcluding } \\
\text { Atchafalaya-Red River } \\
\text { basin)..' } 2\end{array}$ & Louisiana ........................... & 593 & (1) & $\begin{array}{l}1,150 \\
\text { (U.S.-Canada) }\end{array}$ & (1) & 2,340 & (2) & $\begin{array}{l}\text { Mississippi River, Minnesota } \\
\text { (Clearwater County). }\end{array}$ \\
\hline 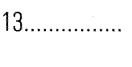 & 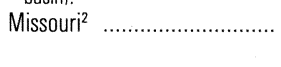 & 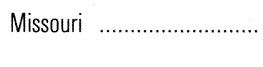 & 76.2 & (6) & $\begin{array}{l}529 \\
\text { (U.S.-Canada) }\end{array}$ & (2) & 2,540 & (1) & $\begin{array}{l}\text { Red Rock Creek, Montana } \\
\text { (Beaverhead County). }\end{array}$ \\
\hline $14 \ldots \ldots \ldots \ldots \ldots . . . .$. & 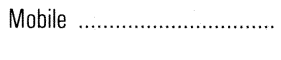 & Alabama ........................... & 67.2 & (8) & 44.6 & $\ldots$ & 774 & (20) & $\begin{array}{l}\text { Tickanetley Creek, Georgia } \\
\text { (Gilmer County). }\end{array}$ \\
\hline 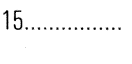 & North Canadian .................... & 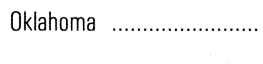 & $\left|{ }^{*}\right|$ & $\cdots$ & 17.6 & $\cdots$ & 800 & (19) & $\begin{array}{l}\text { Corrumpa Creek, New Mexico } \\
\text { (Union County). }\end{array}$ \\
\hline $16 \ldots \ldots \ldots \ldots . . .$. & 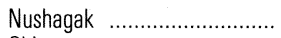 & 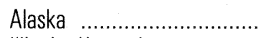 & 36 & (20) & 13.4 & $\cdots$ & 285 & $\cdots$ & Nushagak River, Alaska. \\
\hline $17 \ldots \ldots \ldots \ldots \ldots . . .$. & 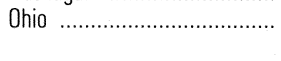 & Illinois-Kentucky ................. & 281 & (3) & 203 & (8) & 1,310 & $|9|$ & $\begin{array}{l}\text { Allegheny River, Pennsylvania } \\
\text { (Potter County). }\end{array}$ \\
\hline $18 \ldots \ldots \ldots \ldots . . . . .$. & 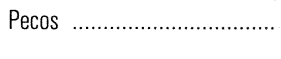 & 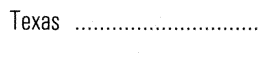 & $(*)$ & $\cdots$ & 44.3 & $\cdots$ & 926 & (15) & $\begin{array}{l}\text { Pecos River, New Mexico } \\
\text { (Mora County). }\end{array}$ \\
\hline 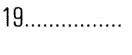 & Platte & Nebraska .......................... & $|*|$ & $\cdots$ & 84.9 & $(13)$ & 990 & (14) & $\begin{array}{l}\text { Grizzly Creek, Colorado } \\
\text { (Jackson County). }\end{array}$ \\
\hline 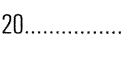 & 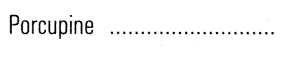 & 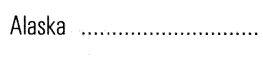 & 23 & $\cdots$ & $\begin{array}{l}45.1 \\
\text { (U.S.-Canada) }\end{array}$ & $|20|$ & 569 & $\ldots$ & $\begin{array}{l}\text { Porcupine River, Yukon Territory, } \\
\text { Canada. }\end{array}$ \\
\hline 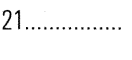 & $\operatorname{Red}^{1} \ldots \ldots$ & 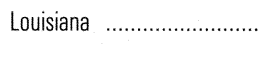 & 56.0 & (13) & 93.2 & $(12)$ & 1,290 & (10) & $\begin{array}{l}\text { Tierra Blanca Creek, New Mexico } \\
\text { (Curry County). }\end{array}$ \\
\hline $22 \ldots \ldots \ldots \ldots \ldots$ & 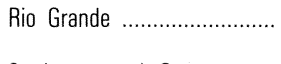 & 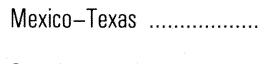 & $|*|$ & $\cdots$ & $\begin{array}{l}336 \\
\text { (U.S.-Mexico) }\end{array}$ & $|4|$ & 1,900 & (4) & $\begin{array}{l}\text { Rio Grande, Colorado } \\
\text { (San Juan County). }\end{array}$ \\
\hline $23 \ldots \ldots \ldots \ldots \ldots$ & $\begin{array}{l}\text { St. Lawrence (-Great } \\
\text { Lakes). }\end{array}$ & 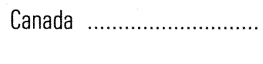 & 348 & (2) & $\begin{array}{l}396 \\
\text { (U.S.-Canada) }\end{array}$ & (3) & 1,900 & (4) & $\begin{array}{l}\text { North River, Minnesota } \\
\text { (Lake County). }\end{array}$ \\
\hline 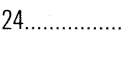 & 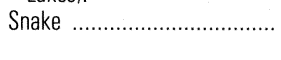 & Washington .......................... & 56.9 & (12) & 108 & $(10)$ & 1,040 & (13) & $\begin{array}{l}\text { Snake River, Wyoming } \\
\text { (Teton County). }\end{array}$ \\
\hline $25 \ldots \ldots \ldots \ldots \ldots$ & Stikine & Alaska ................................ & 56 & $|13|$ & (U.S.-Canada) & $\cdots$ & 379 & $\ldots$ & $\begin{array}{l}\text { Stikine River, British Columbia } \\
\text { Canada. }\end{array}$ \\
\hline $26 \ldots \ldots \ldots \ldots \ldots$ & 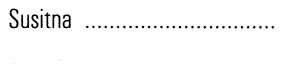 & Alaska ............................... & 51 & (15) & 20 & $\cdots$ & 313 & $\cdots$ & $\begin{array}{l}\text { Susitna River at terminus of } \\
\text { Susitna Glacier, Alaska. }\end{array}$ \\
\hline $27 \ldots \ldots \ldots \ldots \ldots$ & 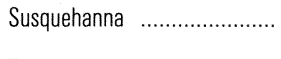 & 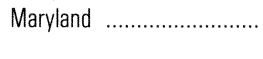 & 38.2 & (18) & 27.2 & $\cdots$ & 447 & $\ldots$ & $\begin{array}{l}\text { Hayden Creek, New York } \\
\text { (Otsego County). }\end{array}$ \\
\hline $28 \ldots \ldots \ldots \ldots \ldots$ & 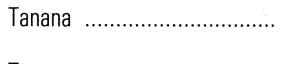 & 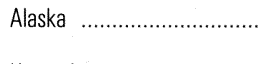 & 41 & $(16)$ & 44.5 & $\cdots$ & 659 & $\cdots$ & $\begin{array}{l}\text { Nabesna River at terminus of } \\
\text { Nabesna Glacier, Alaska. }\end{array}$ \\
\hline 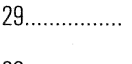 & Tennessee & 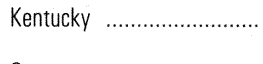 & 68.0 & (7) & 40.9 & $\cdots$ & 886 & (17) & $\begin{array}{l}\text { Courthouse Creek, } \\
\text { North Carolina (Transylvania County). }\end{array}$ \\
\hline 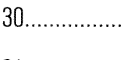 & 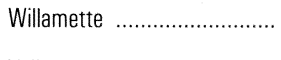 & Oregon & 37.4 & (19) & 11.4 & $\ldots$ & 309 & $\cdots$ & $\begin{array}{l}\text { Middle Fork Willamette River, Oregon } \\
\text { (Douglas County). }\end{array}$ \\
\hline 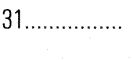 & Yellowstone .......................... & North Dakota ..................... & $\left({ }^{*}\right)$ & $\cdots$ & 70.0 & $(14)$ & 692 & $\cdots$ & $\begin{array}{l}\text { North Folk Yellowstone River, Wyoming } \\
\text { (Park County). }\end{array}$ \\
\hline 32.................. & Yukon & 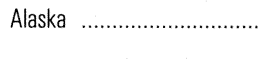 & 225 & (5) & $\begin{array}{l}328 \\
\text { (U.S.-Canada) }\end{array}$ & (5) & 1,980 & (3) & $\begin{array}{l}\text { McNeil River, Yukon Territory, } \\
\text { Canada. }\end{array}$ \\
\hline
\end{tabular}

*Less than $15,000 \mathrm{ft}^{3} / \mathrm{s}$, and therefore not among the largest rivers in terms of discharge.

IIn east-central Louisiana 50 miles northwest of Baton Rouge, the Red River flows into the Atchafalaya River, a distributary of the Mississippi River. The discharge of the Atchafalaya River, as shown in the table above, includes the entire discharge of the Red River, but excludes all water diverted into the Atchafalaya River from the Mississippi River. Thus, the respective discharges represent drainage from corresponding drainage areas. ${ }^{2}$ The total discharge from the entire 1,250,000-mi Mississippi River system, including the Atchafalaya, Red, and Missouri River basins, averages 651,000 cubic feet per second. For the Mississippi River system as a whole, the longest continuous river channel is from the Missouri River headwater source in Montana to the mouth of the Missouri to the Gulf of Mexico, a combined length of about 3,710 miles. 